\section{Pacifier use as a risk factor for reduction in breastfeeding duration: a systematic review}

\section{Uso de chupeta como fator de risco para o tempo de amamentação: uma revisão sistemática}

Edson Theodoro dos Santos Neto 1

Adauto Emmerich Oliveira 2

Eliana Zandonade 3

Maria del Carmem Bisi Molina 4

\section{Abstract}

This systematic review of literature proposes to establish whether pacifier use is a risk factor for a reduction in the duration of breastfeeding. A search of the Medline and Lilacs databases was carried out for articles published between 1996 and 2006 using the following descriptors: "breastfeeding" and "pacifier". Articles were excluded if they: had no open access abstracts; did not contain estimators testing the degree of association between breastfeeding duration and pacifier use; involved a follow-up loss of greater than 20\%; were written in languages other than Portuguese, English and Spanish; covered specific population categories, such as pre-term babies or mothers who experienced difficulty breastfeeding; or were based on information provided by the mother or a health care professional. Nineteen articles were chosen according to the criteria outlined above; of these, one was a randomized clinical trial, eleven were prospective cohort studies and seven were cross sectional studies. The review concludes that pacifier use is a risk factor for breastfeeding duration, although the performance mechanism remains obscure. Other risk factors relate to the children, their parents and the hospital care they receive regarding the early interruption of breastfeeding. There is a need to standardize the methodology for investigating the association between pacifier use and breastfeeding duration, in order to provide scientific knowledge on this subject.

Key words Breastfeeding, Pacifiers, Weaning

\section{Resumo}

Esta revisão sistemática da literatura se propõe verificar se o uso de chupeta é um fator de risco para redução do tempo de amamentação. Foram pesquisadas as bases de dados Medline e Lilacs, no período de 1996 a 2006, utilizando os termos: "aleitamento materno" e "chupeta”, “amamentação". Foram excluidos artigos: sem resumo; sem descrição ou referência a estimadores de associação entre tempo de amamentação e uso de chupetas; com o indice de perda amostral superior a 20\%; escritos em outros idiomas diferentes do português, inglês e espanhol; com abordagem à população de bebês prematuros ou à mães com dificuldades de amamentação; e, com informações relatadas por outros indivíduos diferentes da mãe ou profissional de saúde. Foram selecionados 19 artigos: um ensaio clínico randomizado controlado, 11 estudos de coorte e sete estudos transversais. Conclui-se que uso de chupeta é um fator de risco para a redução do tempo de amamentação, entretanto permanece obscuro qual o seu mecanismo de atuação. Outros fatores de risco relacionados à criança, à mãe, ao pai e ao atendimento hospitalar também influenciam nesse processo. É necessário a padronização de elementos metodológicos que investiguem a associação entre o uso de chupetas e a duração da amamentação visando sintetizar o conhecimento cientifico.

Palavras-chave Aleitamento materno, Chupetas, Desmame 


\section{Introduction}

Breastfeeding duration is an important predictor of infant health and this is supported by the knowledge that through breastfeeding the newborn receives the most appropriate and complete nourishment: breast milk. Acknowledging its importance, the World Health Organization (WHO) ${ }^{1}$ released in 2001 the results of an extended systematic review recommending, in short, that the minimum duration for exclusive breastfeeding should be six months, after which time the newborn should be fed mother's milk and complementary foods until over two years old. During this period, several other factors that can influence breastfeeding duration need to be taken into consideration; these factors range from the mother's characteristics and attitude, attributes pertaining to the father and the newborn and healthcare assistance, and other political, cultural and socioeconomic determinants.

Pacifier use and its relation to breastfeeding duration have been in the spotlight ever since Victora et al. 2 first published an article in an international journal associating breastfeeding duration with the use of a pacifier. This article examines studies of the repercussions of pacifier use on child health.

However, pacifier use is now considered a protective factor for sudden infant death syndrome, ${ }^{3}$ although this association is not that well established owing to the low incidence of this condition and the lack of a clear understanding of its physiological basis. 4 Other authors have preferred to investigate the analgesic effect of the pacifier on babies that have experienced postpartum complications, claiming that sucking has an effect on physiological pain mechanisms, stimulating the pleasure areas and inhibiting pain receptor pathways. ${ }^{5}$

The harmful effects of pacifier use on children's health have also been associated with early weaning, which by itself, can lead to serious child health complications. The child no longer receives the immune protection provided by breast milk and is therefore more vulnerable to intestinal and respiratory infections. ${ }^{6}$ It is known that these infections can easily worsen to the point where they have an effect on infant mortality indicators. ${ }^{7}$ Furthermore, when breastfeeding is replaced by bottle-feeding for example, the child is denied the psychological benefits of direct contact with the mother, being led to develop a totally artificial relation to an object. It is also argued that the pacifier could, by diminishing a child's crying, conceal serious health issues. ${ }^{8}$

Another main consequence of the long-term use of pacifier is the effect on dental occlusion. It has frequently been associated with anterior open bite, crossbite and important musculoskeletal alterations. 9 Sucking the pacifier is supposed to act as a functional matrix that misguides the growth and development of the facial bones and muscles. ${ }^{10}$ The aesthetic and functional consequences may last a lifetime, limiting the individual's ability to chew, his or her respiratory and phonetic capacity, as well as causing disfiguration.

Given its known significance, the BabyFriendly Hospital Initiative, recommends, as its 9th step towards successful breastfeeding, that pacifier use be discouraged among breastfed children. Likewise, Brazilian Law 11,265 of January 3 rd, 2006, concerning the sale of infant formulas, baby food and other child-care related products, requires that companies display on the labels of these products a warning that pacifier use is harmful to children's health in many ways. 11 Still, there are no studies that bring together the findings of the literature and it is thus not possible to affirm that pacifier use really does have a detrimental effect on the duration of breastfeeding.

In view of the facts described above, this systematic review of the literature aims to establish how the relation between pacifier use and breastfeeding duration is presented in the scientific literature on the subject.

\section{Methods}

Articles addressing the association between breastfeeding and pacifier use published between 1996 and 2006 were reviewed. To do this, the Medline and Lilacs databases were searched for the key words "breastfeeding" and "pacifier" in both Portuguese and English.

This first phase excluded all the articles without open access abstracts or written in languages other than Portuguese, English and Spanish, along with those whose abstracts did not present estimators testing the association between breastfeeding duration and pacifier use. On Lilacs a total of 18 articles and one thesis were found; of these, 11 did not include estimators because they focused on legislation, oral health and women's health. Seven articles were thus selected to be read in full. The thesis was 
excluded because there was a corresponding article available.

On Medline, 43 articles were found in Portuguese dating from between 1996 and 2006. Thirty-one were excluded, either because they did not cite estimators, focused on oral health, sudden death syndrome, women's health or pain, or because they were already review articles, were written in German, or did not have an abstract. The second search performed on Medline, this time using the keywords in English, yielded 84 articles. Forty-two of these did not provide references of association between the variables of interest; two did not have abstracts; four were written in other languages such as German, Polish and Danish. After all the exclusions, this second Medline search yielded 36 articles, which were added to the seven found on Lilacs and the ten yielded by the first Mediline. A total of 46 articles were therefore selected to be read in full. Analysis of these 46 articles led to the exclusion of a further 18 , because they appeared on more than one database. The study finally included therefore a total of 26 articles.

Most of the selected articles are available online at CAPES Portal, when accessing through a federal institution. The authors of articles unavailable online were contacted by e-mails asking for the articles of interest. The Network of Federal Libraries in Brazil was used to recover articles. Thirty-three complete articles were read. These were classified as follows: five randomized and controlled clinical trials, two case-control studies, 18 cohort studies, and eight cross-sectional studies.

To be included in the analysis the articles had to perform a direct association between exclusive breastfeeding duration and pacifier use or breastfeeding and pacifier use; the information regarding this must have been provided by the mother or a health care professional; the follow up loss could not be greater than $20 \%$; the studies could not focus on specific population categories such as pre-term babies or mothers who had trouble with breastfeeding, because these kinds of groups usually get treated differently and this can interfere as a confounding factor in the study. These criteria were based on Ullah \& Griffiths ${ }^{12}$ systematic review.

The study performed by Görbe et al.13 was excluded because it did not show an adequate association between breastfeeding at the sixth month and pacifier use but merely described that, in a group of 136 non-pacifier users, eight stopped breastfeeding before the sixth month.
The studies performed by Levy et al.14 and Marques et al. 15 were excluded because of their follow-up losses. In the first one, this was $24 \%$ for the first six months of life and, in the second, $21 \%$ for the first year. Binns \& Scott's study 16 presents an initial sample of 556 and this was also excluded from the evaluation because it presents a table with interest variables for a sample size of only 372 mother-infant pairs, suggesting a follow-up loss of $33.1 \%$.

Baker's ${ }^{17}$ study was also excluded due to the fact that it does not display details regarding its methods and inclusion criteria, which ruled out evaluation according to the criteria described above. Righard 18 and Righard and Alade's 19 samples involved only mothers who had trouble breastfeeding, mainly due to ulcers, congestion and breast infections and both studies did not therefore stand up to the inclusion criteria. The study conducted by Kloeblen-Tanver 20 was excluded for the same reason and for suggesting that $84 \%$ of its sample consisted of mothers and children undergoing a special nutrition supplementation program. The randomized clinical trial of Centuori et al.21 could not be used because it was testing a medication that aimed to prevent breast diseases, which are considered limiting factors for breastfeeding duration.

The study of Barros et al.22 was excluded for testing an association between birth weight and pacifier use as well as birth weight and breastfeeding duration and therefore not testing the variables of interest for this review; the study carried out by Kramer et al..8 was also excluded because it tested the effects of counseling mothers on breastfeeding, thereby discouraging the use of a pacifier and not testing it as a predictor of breastfeeding duration.

The study of Collins et al. ${ }^{23}$ used a sample of pre-term babies; Cunha et al. ${ }^{24}$ were also excluded for including children whose birth weight was under three kilos and healthy, which suggested that the greater part of this sample consisted of pre-term babies, thereby restricting the possibility of interpreting the results.

Dewey et al.'s 25 study could not be included in the analysis either, because it did not make a direct association between pacifier use and breastfeeding duration, despite associating the early use of pacifier by mothers who received health care for one week only.

Even though Hornell et al.'s26 study conducted a partial analysis of the data set also used by Aarts et $a l ., 27$ which was included in the evaluation, it had to 
be excluded, since it did not perform a direct association between exclusive breastfeeding duration and pacifier use or breastfeeding and pacifier use.

Likewise the article by Kelmanson 28 was excluded because it did not establish a relation between breastfeeding duration and pacifier use, even though it shows a statistically significant relation between a pattern of exclusive breastfeeding, formula feeding and bottle feeding with pacifier use.

\section{Results}

Nineteen articles were finally chosen to be included: one randomized and controlled clinical trial, nine cohort studies and eight cross-sectional studies. Table 1 presents brief descriptions of the selected articles.

Most of the studies had no follow-up loss and claim that they had to make exclusions along the way to get to their final sample. These exclusions were not due to follow-up loss but resulted from the inclusion and exclusion criteria of each study.

The minimum follow-up duration was three months and the maximum was 12 months. As for the cross sectional studies, they grouped together children aged from four months until two years of age. The largest sample size was found in MikielKostyra et al.,29 who studied 11,422 mother-infant pairs, while Santiago et al. 30 used the smallest - only 101.

Telephone interviews were used by most of the cohort studies, even though personal contact at the hospital and home visits had an important impact on the quality of information. Recall diaries were also used to confirm information and train researchers and or health professionals. The structured interviews provide evidence of the authors' commitment to ensuring good quality data collection. Table 2 presents the studies according to patterns of breastfeeding.

Sixteen articles found a direct association between breastfeeding duration and pacifier use, ten found a direct association between exclusive breastfeeding and pacifier use, and three articles considered full breast feeding and pacifier use. It was observed that the variables of interest did not establish a pattern for the answers, which could be either qualitative binomial or ordinal qualitative, or even nominal in the case of several categories.
In Aarts et al.27 and Nelson et al.31 chisquare tests and risk values were recalculated according to the sample sizes described in tables, because the categories appearing in the results did not allow a precise relationship between pacifier use and breastfeeding categories to be inferred. Therefore, classification according to the number of individuals per variable was possible. "Exclusive breastfeeding" and "breastfeeding" and "pacifier use" were dichotomized for the purposes of analysis.

Among the methods of statistical analysis, Survival Analysis and Cox Regression prevailed. As a consequence, the most commonly used risk estimation was the Hazards Ratio adjusted for other possible confounding factors. Only Cotrim et al.,32 Vieira et al.33 and Vieira et al.34 did not control for confounding factors.

The confounding factors that remained statistically significant $(p<0.05)$ after a multivariate analysis are also presented in Table 3 . This shows that these factors are also associated to the duration of breastfeeding. Although they attenuate the effects of pacifier use over duration of breastfeeding, the effect is not completely canceled out. To facilitate visualization, the studies were distributed according to variable type, in so far as it relates to the child, the mother, the father or the place of birth.

Among the variables relating to the child, the one that showed greater impact on the reduction of breastfeeding duration was the use of complimentary foods such as a formula or milk in a glass or a bottle.35-40 In addition, the level of education of the mother27,29,31,37,41 and the age of the mother,29,31,36,39,41-43 as well as smoking during pregnancy29,31,42,43 and returning to work $42-44$ had a significant impact on the duration of breastfeeding. The level of education of the father seems to be important for the duration of breastfeeding owing to the fact that a father's support for breastfeeding practices apparently extends its duration. $29,43,45$

The variables relating to hospital care appear to be critical, since hospitals encourage breastfeeding, but most of the selected articles did not control these variables making it impossible to determine whether hospital care influences the duration of breastfeeding or not. 
Table 1

Categorization of studies according to study desingn.

\begin{tabular}{|c|c|c|c|c|}
\hline Clinical trial & Sample (n) & Country & Follow-up duration & Methods of data collection \\
\hline Howard et al., 2003.35 & 700 & USA & 52 weeks ( 12 months) & $\begin{array}{l}\text { Phone interviews carried out by nurses in } \\
\text { the } 2^{\text {nd }}, 5^{\text {th }}, 10^{\text {th }}, 16^{\text {th }} \text {, and } 24^{\text {th }}, 38^{\text {th }} \\
\text { and } 52^{\text {nd }} \text { post-partum weeks. }\end{array}$ \\
\hline \multicolumn{5}{|l|}{ Cohort } \\
\hline Victora et al., 1997.36 & 650 & Brazil & 6 months & $\begin{array}{l}\text { Data from hospital and structured } \\
\text { interviews carried out by researchers } \\
\text { during home visits in the } 3^{\text {rd }} \text { e } 6 \text { th post- } \\
\text { partum months }\end{array}$ \\
\hline Howard et al., 1999.44 & 311 & USA & 24 weeks ( 6 months) & $\begin{array}{l}\text { Phone interviews carried out by nurses in } \\
\text { the } 2^{\text {nd }}, 6^{\text {th }}, 12^{\text {th }} 24^{\text {th }} \text { post-partum } \\
\text { weeks. }\end{array}$ \\
\hline Aarts et al., 1999.27 & 430 & Sweden & Mean $=8.6$ (months) $\pm 3.4 \mathrm{dp}$ & $\begin{array}{l}\text { Mother's recall diaries and structured } \\
\text { interviews carried out by research } \\
\text { assistants during home visits }\end{array}$ \\
\hline Riva et al., 1999.37 & 1365 & Italy & 12 months & 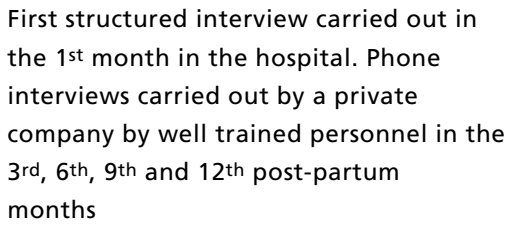 \\
\hline Vogel et al., 2001. 41 & 350 & New Zealand & 12 months & $\begin{array}{l}\text { First structured interview carried out at } \\
\text { home and by phone in the } 1 \text { st, } 2^{\text {nd }}, 3 \text { rd, } \\
6^{\text {th }} \text { e } 12^{\text {th }} \text { months by trained researchers } \\
\text { using a pre-coded questionnaire. }\end{array}$ \\
\hline Santiago et al.,2003.30 & 101 & Brazil & 4 months & $\begin{array}{l}\text { Data collected from standard clinical files, } \\
\text { filled out by three pediatricians. }\end{array}$ \\
\hline Soares et al., 2003.38 & 250 & Brazil & 6 months & $\begin{array}{l}\text { First structured interview carried out at } \\
\text { the hospital, and home visits in the } 1 \text { st } \\
\text { and } 6 \text { th months; and, phone interviews in } \\
\text { the } 2^{\text {nd }} \text { and } 4 \text { th post-partum months. All } \\
\text { performed by well trained medical } \\
\text { students. }\end{array}$ \\
\hline Giovannini et al., 2004.39 & 2450 & Italy & 12 months & $\begin{array}{l}\text { carried out in the } 1 \text { st month in the } \\
\text { hospital and using Mother's recall } \\
\text { diaries. Phone interviews carried out by a } \\
\text { private company by well trained } \\
\text { personnel in the } 3 \text { rd, } 6 \text { th, } 9 \text { th and } 12^{\text {th }} \\
\text { post-partum months }\end{array}$ \\
\hline Nelson et al., 2005.31 & 2844 & 17 countries & $\begin{array}{l}10 \text { to } 14 \text { weeks and } \\
3 \text { at } 6 \text { months }\end{array}$ & $\begin{array}{l}\text { First structured interview at birth, home } \\
\text { questionnaire posted } 12 \text { weeks after birth } \\
\text { with questions referring to "last night." } \\
\text { face-to-face interviews, and others used } \\
\text { retrospectively to collect birth } \\
\text { information data. }\end{array}$ \\
\hline
\end{tabular}


Categorization of studies according to study desingn

\begin{tabular}{|c|c|c|c|c|}
\hline Cohort & Sample (n) & Country & Follow-up duration & Methods of data collection \\
\hline Scott et al., 2006.42 & 587 & Australia & 52 weeks ( 13 months) & $\begin{array}{l}\text { First self-explanatory questionnaire, } \\
\text { home visit three days after hospital } \\
\text { discharge, structured phone interview in } \\
\text { the } 4 \text { th, } 10^{\text {th }, ~} 16^{\text {th }, ~} 22^{\text {nd }}, 32 \text { nd, } 40 \text { th, } \\
\text { and } 52 \text { nd weeks. }\end{array}$ \\
\hline Mascarenhas et al., 2006.43 & 973 & Brazil & 3 months & $\begin{array}{l}\text { First structured interview in the } 1 \text { st } \\
\text { month at the hospital of birth and home } \\
\text { visit in the 3rd month carried out by } \\
\text { medical students. }\end{array}$ \\
\hline \multicolumn{5}{|l|}{ Cross-Sectional } \\
\hline Mikiel-Kostyra et al., 2002.29 & 11.422 & Poland & Less than 6 months old & Analysis of data sets from Polish hospitals \\
\hline Cotrim et al., 2002.32 & 22.188 & Brazil & Less than 4 months old & $\begin{array}{l}\text { Analysis of data set assembled by health } \\
\text { professionals during Brazil's National } \\
\text { Vaccination Campaign. }\end{array}$ \\
\hline Audi et al., 2003.46 & $\begin{array}{l}679 \\
346\end{array}$ & $\begin{array}{l}\text { Brazil } \\
\text { Brazil }\end{array}$ & $\begin{array}{l}\text { Less than } 1 \text { year old' } \\
\text { Less than } 6 \text { months old }\end{array}$ & $\begin{array}{l}\text { Structured form applied by } 20 \text { trained } \\
\text { health professional and six nurses during } \\
\text { Brazil's National Vaccination Campaign. }\end{array}$ \\
\hline Vieira et al., 2004.34 & $1912 *$ & Brazil & Less than 1 year old & $\begin{array}{l}\text { Data collected on Brazil's National } \\
\text { Vaccination Campaign by } 104 \text { trained } \\
\text { university students on health-related } \\
\text { courses. }\end{array}$ \\
\hline Minagawa et al., 2005.40 & 261 & Brazil & Less than 2 years old & $\begin{array}{l}\text { Home-based data collection, carried out } \\
\text { by researchers using structured } \\
\text { questionnaires. }\end{array}$ \\
\hline Silveira e Lamounier, 2006.45 & 450 & Brazil & Less than 2 years old & $\begin{array}{l}\text { Structured interview carried out by } \\
\text { medical students in community health } \\
\text { centers, and homes in a specific region. }\end{array}$ \\
\hline
\end{tabular}

* Both studies used a sub-sample to check the association between pacifier use and breastfeeding duration. 
Table 2

Breastfeeding duration versus pacifier use.

\begin{tabular}{|c|c|c|c|c|c|c|}
\hline Article & Outcome & $\begin{array}{l}\text { Variables of } \\
\text { interest }\end{array}$ & $\begin{array}{l}\text { Statistical } \\
\text { analysis }\end{array}$ & $p$-value & Risk estimator & $\begin{array}{c}\text { Confidence } \\
\text { Interval (95\%) }\end{array}$ \\
\hline $\begin{array}{l}\text { Howard et al. } \\
2003.35\end{array}$ & Breastfeeding & $\begin{array}{l}\text { Pacifier use up to } 1 \text { st } \\
\text { month: Yes } x \text { No }\end{array}$ & $\begin{array}{l}\text { Survival analysis } \\
\text { Cox regression }\end{array}$ & 0.020 & Hazard ratio (adjusted) $=1.22$ & $1.03-1.44$ \\
\hline $\begin{array}{l}\text { Howard et al. } \\
2003.35\end{array}$ & $\begin{array}{l}\text { Full } \\
\text { breastfeeding }\end{array}$ & $\begin{array}{l}\text { Pacifier use up to } 1 \text { st } \\
\text { month: Yes } x \text { No }\end{array}$ & $\begin{array}{l}\text { Survival analysis } \\
\text { Cox regression }\end{array}$ & 0.640 & Hazard ratio (adjusted) $=1.04$ & $0.89-1.21$ \\
\hline $\begin{array}{l}\text { Victora et al. } \\
1997.36\end{array}$ & Breastfeeding & $\begin{array}{l}\text { Daily pacifier use from } 1 \text { st } \\
\text { to 6th month: } \\
\text { Partially } \\
\text { Always } \\
\text { Never }\end{array}$ & $\begin{array}{l}\text { Survival analysis } \\
\text { Cox regression }\end{array}$ & $<0.001$ & $\begin{array}{l}\text { Hazard ratio (adjusted) } \\
1.74 \\
2.37 \\
1.0\end{array}$ & $\begin{array}{l}1.15-2.63 \\
1.40-4.01\end{array}$ \\
\hline $\begin{array}{l}\text { Howard et al. } \\
1999.44\end{array}$ & Breastfeeding & $\begin{array}{l}\text { Pacifier use on the first } 6 \\
\text { weeks: Yes } x \text { No }\end{array}$ & $\begin{array}{l}\text { Survival analysis } \\
\text { Cox regression }\end{array}$ & 0.004 & Hazard ratio (adjusted) $=1.53$ & $1.15-2.05$ \\
\hline $\begin{array}{l}\text { Howard et al. } \\
1999.44\end{array}$ & $\begin{array}{l}\text { Full } \\
\text { breastfeeding }\end{array}$ & $\begin{array}{l}\text { Pacifier use on the first } 6 \\
\text { weeks: Yes } x \text { No }\end{array}$ & $\begin{array}{l}\text { Survival analysis } \\
\text { Cox regression }\end{array}$ & 0.002 & Hazard ratio (adjusted) $=1.61$ & $1.19-2.19$ \\
\hline $\begin{array}{l}\text { Aarts et al. } \\
1999.27\end{array}$ & Breastfeeding & $\begin{array}{l}\text { Pacifier use up to } 4^{\text {th }} \\
\text { month: } \\
\text { Never } \\
\text { Occasionally } \\
\text { Always } \\
\text { Frequently }\end{array}$ & $\begin{array}{l}\text { Survival analysis } \\
\text { Cox regression }\end{array}$ & $<0.05$ & $\begin{array}{l}\text { Hazard ratio (adjusted)= } \\
1.0 \\
1.7 \\
1.62 \\
2.17\end{array}$ & $\begin{array}{l}0.79-1.47 \\
1.28-2.07 \\
1.53-3.09\end{array}$ \\
\hline $\begin{array}{l}\text { Riva et al. } \\
1999.37\end{array}$ & Breastfeeding & $\begin{array}{l}\text { Pacifier use up to } 1 \text { st } \\
\text { month: } \\
\text { Yes } x \text { No }\end{array}$ & $\begin{array}{l}\text { Survival analysis } \\
\text { Cox regression }\end{array}$ & $=0.008$ & Rate ratio (adjusted) $=1.18$ & $1.04-134$ \\
\hline $\begin{array}{l}\text { Vogel et al. } \\
2001.41\end{array}$ & Breastfeeding & $\begin{array}{l}\text { Daily pacifier use up to } 1 \text { st } \\
\text { month: Yes } x \text { No }\end{array}$ & $\begin{array}{l}\text { Multiple logistic } \\
\text { regression }\end{array}$ & $?$ & Relative risk $=1.35$ & $1.05-1.74$ \\
\hline $\begin{array}{l}\text { Soares et al. } \\
2003.38\end{array}$ & Breastfeeding & $\begin{array}{l}\text { Pacifier use up to } 6 \text { th } \\
\text { month: Yes } x \text { No }\end{array}$ & $\begin{array}{l}\text { Survival analysis } \\
\text { Cox regression }\end{array}$ & $<0.01$ & $\begin{array}{l}\text { Incidence density ratio } \\
\text { (adjusted) }=2.2\end{array}$ & $1.3-3.8$ \\
\hline $\begin{array}{l}\text { Giovanini et } \\
\text { al.. 2004.39 }\end{array}$ & Breastfeeding & Up to 1 st month: & $\begin{array}{l}\text { Survival analysis } \\
\text { Cox regression }\end{array}$ & $<0.001$ & Hazard ratio (adjusted) $=1.30$ & $1.15-1.46$ \\
\hline $\begin{array}{l}\text { Nelson et al. } \\
2005.31\end{array}$ & Breastfeeding & $\begin{array}{l}\text { Pacifier use most of time } \\
\text { and short time: Yes } x \text { No }\end{array}$ & $\begin{array}{l}\text { Univariate } \\
\text { analysis }\end{array}$ & 0.000 & $\begin{array}{l}\text { Relative Risks=1.38 } \\
\text { Odds ratio }=2.160\end{array}$ & $\begin{array}{l}1.30-1.47 \\
1.85-2.53\end{array}$ \\
\hline $\begin{array}{l}\text { Scott et al. } \\
2006.42\end{array}$ & Breastfeeding & $\begin{array}{l}\text { Pacifier use: } \\
\text { Up to the } 4 \text { th week } \\
\text { From } 4 \text { th to } 10 \text { th week } \\
\text { After } 10^{\text {th }} \text { week } \\
\text { Didn't use until } 12 \text { months }\end{array}$ & $\begin{array}{l}\text { Survival analysis } \\
\text { Cox regression }\end{array}$ & $<0.05$ & $\begin{array}{l}\text { Hazard ratio (adjusted) } \\
1.92 \\
1.97 \\
1.61 \\
1.0\end{array}$ & $\begin{array}{l}1.40-2.64 \\
1.13-3.46 \\
0.86-3.00\end{array}$ \\
\hline $\begin{array}{l}\text { Cotrim et al. } \\
2002.32\end{array}$ & Breastfeeding & $\begin{array}{l}\text { Pacifier use up to } 4 \text { th } \\
\text { month: Yes } \times \text { No }\end{array}$ & Univariate analysis & $<0.05$ & Odds ratio $=5.99$ & $5.50-6.60$ \\
\hline $\begin{array}{l}\text { Audi et al. } \\
2003.46\end{array}$ & Breastfeeding & $\begin{array}{l}\text { Pacifier use in children } \\
\text { under } 12 \text { months: Yes } x \text { No }\end{array}$ & $\begin{array}{l}\text { Multiple logistic } \\
\text { regression }\end{array}$ & $<0.001$ & Odds ratio $=6.58$ & $3.94-7.89$ \\
\hline $\begin{array}{l}\text { Vieira et al. } \\
2004.34\end{array}$ & Breastfeeding & $\begin{array}{l}\text { Pacifier use up to } 12^{\text {th }} \\
\text { month: Yes } \times \text { No }\end{array}$ & Univariate analysis & $<0.001$ & Prevalence ratio $=1.60$ & $1.39-1.84$ \\
\hline
\end{tabular}


Breastfeeding duration versus pacifier use.

\begin{tabular}{|c|c|c|c|c|c|c|}
\hline Article & Outcome & $\begin{array}{l}\text { Variables of } \\
\text { interest }\end{array}$ & $\begin{array}{l}\text { Statistical } \\
\text { analysis }\end{array}$ & p-value & Risk estimator & $\begin{array}{c}\text { Confidence } \\
\text { Interval }(95 \%)\end{array}$ \\
\hline $\begin{array}{l}\text { Vieira et al. } \\
2004.33\end{array}$ & Breastfeeding & $\begin{array}{l}\text { Pacifier use up to } 4 \text { th } \\
\text { month: Yes } x \text { No }\end{array}$ & $\begin{array}{l}\text { Univariate } \\
\text { analysis }\end{array}$ & $<0.05$ & Prevalence ratio $=1.52$ & $1.36-1.71$ \\
\hline $\begin{array}{l}\text { Minagawa } \\
\text { et al. } 2005.40\end{array}$ & Breastfeeding & $\begin{array}{l}\text { Pacifier use in children } \\
\text { under } 24 \text { months: Yes x } \\
\text { No }\end{array}$ & $\begin{array}{l}\text { Survival analysis } \\
\text { Cox regression }\end{array}$ & $<0.05$ & Hazard ratio (adjusted) $=2.84$ & $1.95-4.19$ \\
\hline $\begin{array}{l}\text { Silveira e } \\
\text { Lamounier. } \\
2006.45\end{array}$ & Breastfeeding & $\begin{array}{l}\text { Pacifier use in children } \\
\text { under } 24 \text { months: Yes x } \\
\text { No }\end{array}$ & $\begin{array}{l}\text { Survival analysis } \\
\text { Cox regression }\end{array}$ & 0.000 & Hazard ratio (adjusted) $=3.16$ & $2.23-4.48$ \\
\hline $\begin{array}{l}\text { Howard et al.. } \\
2003.35\end{array}$ & $\begin{array}{l}\text { Exclusive } \\
\text { breastfeeding }\end{array}$ & $\begin{array}{l}\text { Pacifier use up to } 1 \text { st } \\
\text { month: Yes } x \text { No }\end{array}$ & $\begin{array}{l}\text { Survival analysis } \\
\text { Cox regression }\end{array}$ & $=0.26$ & Hazard ratio (adjusted) $=1.09$ & $0.94-1.27$ \\
\hline $\begin{array}{l}\text { Aarts et al. } \\
\text { 1999. } 27\end{array}$ & $\begin{array}{l}\text { Exclusive } \\
\text { breastfeeding }\end{array}$ & $\begin{array}{l}\text { Pacifier use up to } 4 \text { th } \\
\text { month: Frequently } x \\
\text { Never }\end{array}$ & $\begin{array}{l}\text { Survival analysis } \\
\text { Cox regression } \\
\text { Univariate } \\
\text { analysis \# }\end{array}$ & $\begin{array}{l}=0.009 \\
=0.006\end{array}$ & $\begin{array}{l}\text { Hazard ratio (adjusted) }=? \\
\text { Odds ratio }=1.82\end{array}$ & $\begin{array}{l}3.9-23.8 \\
1.17-2.82\end{array}$ \\
\hline $\begin{array}{l}\text { Riva et al. } \\
1999.37\end{array}$ & $\begin{array}{l}\text { Exclusive } \\
\text { breastfeeding }\end{array}$ & $\begin{array}{l}\text { Pacifier use up to } 1 \text { st } \\
\text { month: Yes } x \text { No }\end{array}$ & $\begin{array}{l}\text { Survival analysis } \\
\text { Cox regression }\end{array}$ & $<0.001$ & Rate ratio (adjusted) $=1.35$ & $\begin{array}{l}3.9-23.8 \\
1.17-2.82\end{array}$ \\
\hline $\begin{array}{l}\text { Santiago et al. } \\
2003.30\end{array}$ & $\begin{array}{l}\text { Exclusive } \\
\text { breastfeeding }\end{array}$ & $\begin{array}{l}\text { Pacifier use up to } 1 \text { st } \\
\text { month: No } x \text { Yes }\end{array}$ & $\begin{array}{l}\text { Multiple logistic } \\
\text { regression }\end{array}$ & $=0.003$ & Odds ratio (adjusted) $=0.23$ & $0.08-0.60$ \\
\hline $\begin{array}{l}\text { Giovanini } \\
\text { et al. } 2004.39\end{array}$ & $\begin{array}{l}\text { Exclusive } \\
\text { breastfeeding }\end{array}$ & $\begin{array}{l}\text { Pacifier use up to } 1 \text { st } \\
\text { month: Yes } x \text { No }\end{array}$ & $\begin{array}{l}\text { Survival analysis } \\
\text { Cox regression }\end{array}$ & $<0.001$ & Hazard ratio (adjusted) $=1.28$ & $1.13-1.45$ \\
\hline $\begin{array}{l}\text { Nelson et al. } \\
2005.31\end{array}$ & $\begin{array}{l}\text { Exclusive } \\
\text { breastfeeding }\end{array}$ & $\begin{array}{l}\text { Pacifier use most of time } \\
\text { and short time: Yes } x \text { No }\end{array}$ & $\begin{array}{l}\text { Univariate analysis } \\
\#\end{array}$ & 0.000 & $\begin{array}{l}\text { Relative risks }=1.61 \\
\text { Odds ratio }=2.13\end{array}$ & $\begin{array}{l}1.46-1.78 \\
1.82-2.50\end{array}$ \\
\hline $\begin{array}{l}\text { Scott et al. } \\
2006.42\end{array}$ & $\begin{array}{l}\text { Full } \\
\text { breastfeeding }\end{array}$ & $\begin{array}{l}\text { Pacifier use: } \\
\text { Up to the } 4 \text { th week } \\
\text { From } 4 \text {. to } 10 \text {. week } \\
\text { After } 10 . \text { week } \\
\text { Didn't use until } 12 \\
\text { months }\end{array}$ & $\begin{array}{l}\text { Survival analysis } \\
\text { Cox regression }\end{array}$ & $<0.05$ & $\begin{array}{l}\text { Hazard ratio (adjusted) } \\
1.92 \\
1.85 \\
1.47 \\
1.0\end{array}$ & $\begin{array}{l}1.39-2.64 \\
1.06-3.22 \\
0.79-2.73\end{array}$ \\
\hline $\begin{array}{l}\text { Mascarenhas } \\
\text { et al. } 2006.43\end{array}$ & $\begin{array}{l}\text { Exclusive } \\
\text { breastfeeding }\end{array}$ & $\begin{array}{l}\text { Pacifier use up to } 3^{\text {rd }} \\
\text { month: Yes } x \text { No }\end{array}$ & $\begin{array}{l}\text { Multivariate } \\
\text { analysis }\end{array}$ & $<0.05$ & Odds ratio (adjusted) $=4.27$ & $3.19-5.72$ \\
\hline $\begin{array}{l}\text { Audi et al. } \\
2003.46\end{array}$ & $\begin{array}{l}\text { Exclusive } \\
\text { breastfeeding }\end{array}$ & $\begin{array}{l}\text { Pacifier use in children } \\
\text { under } 6 \text { months: Yes x No }\end{array}$ & $\begin{array}{l}\text { Multiple logistic } \\
\text { regression }\end{array}$ & $<0.001$ & Odds ratio (adjusted) $=4.41$ & $1.09-2.91$ \\
\hline $\begin{array}{l}\text { Cotrim et al. } \\
2002.32\end{array}$ & $\begin{array}{l}\text { Exclusive } \\
\text { breastfeeding }\end{array}$ & $\begin{array}{l}\text { Pacifier use up to } 4 \text { th } \\
\text { month: Yes } x \text { No }\end{array}$ & $\begin{array}{l}\text { Univariate } \\
\text { analysis }\end{array}$ & $<0.05$ & Odds ratio $=3.26$ & $3.0-3.5$ \\
\hline $\begin{array}{l}\text { Mikiel-Kostyra } \\
\text { et al. } 2005.29\end{array}$ & $\begin{array}{l}\text { Exclusive } \\
\text { breastfeeding }\end{array}$ & $\begin{array}{l}\text { Pacifier use in children } \\
\text { under } 6 \text { months: Yes } x \text { No }\end{array}$ & $\begin{array}{l}\text { Multivariate } \\
\text { analysis }\end{array}$ & $<0.05$ & Odds ratio (adjusted) $=2.38$ & $2.17-2.61$ \\
\hline
\end{tabular}

\# Univariate analysis was performed using $\chi^{2}$.odds ratio and relative risk using the information' described in the tables. 
Significant confounding factors adjusted for the breastfeeding duration.

\begin{tabular}{|c|c|c|c|c|c|c|c|c|c|c|c|c|c|c|c|c|c|}
\hline \multirow[b]{2}{*}{ Confounding factors } & & \multicolumn{16}{|c|}{ Articles } \\
\hline & ling factors & 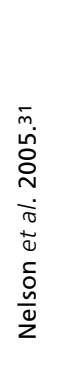 & 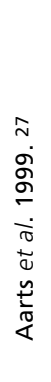 & 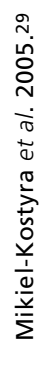 & 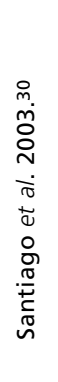 & 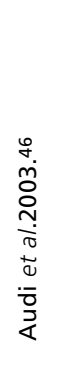 & 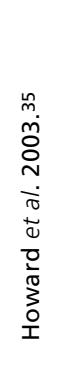 & 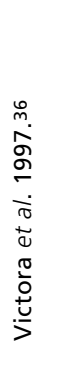 & 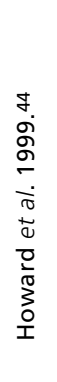 & 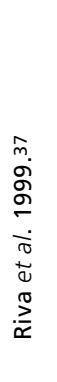 & 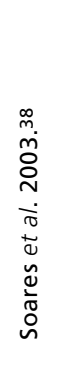 & 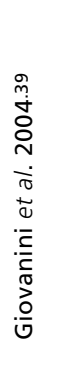 & 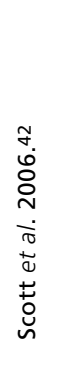 & 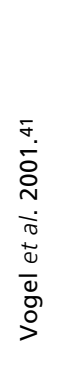 & 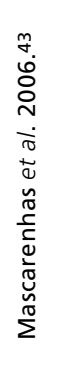 & 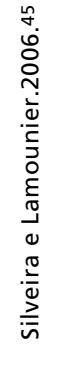 & 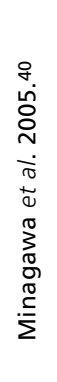 \\
\hline \multirow[t]{6}{*}{ Child } & Sex & & & & & & & & & & & & & $x$ & & & \\
\hline & Birth weight & & & & & $x$ & & $x$ & & & & & & & & & \\
\hline & Birth order & & & $x$ & & & & & & & & & & & & & $x$ \\
\hline & Supplementary feeding using glass or bottle & & & & & & $x$ & $x$ & & $x$ & $x$ & $\mathrm{x}$ & & & & & $x$ \\
\hline & Introduction of solid foods & & & & & & & & & & & $\mathrm{x}$ & & & & & \\
\hline & Child's weight at one month & & & & & & & & & & & $\mathrm{x}$ & & & & & \\
\hline \multirow[t]{14}{*}{ Mother } & Age & & $x$ & $x$ & & & & $x$ & $x$ & & & $\mathrm{x}$ & $x$ & $x$ & $x$ & & \\
\hline & Education & $\mathrm{x}$ & $x$ & $x$ & & & & & & $x$ & & & & $x$ & & & \\
\hline & Skin color & & & & & & & $x$ & & & & & & & & & \\
\hline & Body Mass Index & & & & & & & & & & & $\mathrm{x}$ & & & & & \\
\hline & Smoking during pregnancy & $\mathrm{x}$ & & $x$ & & & & & & & & & $x$ & & $x$ & & \\
\hline & Type of delivery & & & & & $x$ & & $x$ & & & & & & & & & \\
\hline & Parity & $x$ & & & & & & & & & & & & $x$ & & & \\
\hline & Willingness to breastfeed & $x$ & & & & & & & $x$ & & & & $x$ & & & & \\
\hline & Difficulties with breastfeeding in 1st month & & & & & & & $x$ & & & & & $\mathrm{x}$ & & & & \\
\hline & Self-confidence regarding breastfeeding & & & & & & & & & & & & & $x$ & & & \\
\hline & Knowledge about breastfeeding & & & $x$ & & & & & & & & & & & & & \\
\hline & Knowledge about pacifier effects & & & & & & & $x$ & & & & & & & & & \\
\hline & Work status & & & $x$ & & & & & & & & & & & & & \\
\hline & Return to work & & & & & & & & $\mathrm{x}$ & & & & $\mathrm{x}$ & & $x$ & & \\
\hline \multirow[t]{3}{*}{ Father } & Education & & & $x$ & & & & & & & & & & & $x$ & $x$ & \\
\hline & Present in household & & & & & & & & & & & & & & & $\mathrm{x}$ & \\
\hline & Employment status & & & $x$ & & & & & & & & & & & & & \\
\hline \multirow[t]{5}{*}{ Hospital } & Breastfeeding on discharge & & & & & & & $\mathrm{x}$ & & & & & & & & & \\
\hline & Special support from multidisciplinary crew & & & & $x$ & & & & & & & & & & & & \\
\hline & Special support of trained pediatrician & & & & $\mathrm{x}$ & & & & & & & & & & & & \\
\hline & Infant bed shared during time of home & $x$ & & & & & & & & & & & & & & & \\
\hline & Kind of hospital: public/private & & & & & $x$ & & & & & & & & & & & \\
\hline
\end{tabular}




\section{Discussion}

The results indicate that the use of a pacifier has been considered a risk factor for reduction of breastfeeding duration. Only Howard et al.35 did not present significant values when testing the association between pacifier use during the first month of life and exclusive breastfeeding duration (HRadj=1.09; 95\%CI=0.94-1.27). Meanwhile, Santiago et al. ${ }^{30}$ make the opposite claim that non use of pacifier is a protective factor against the reduction of the duration of exclusive breastfeeding.

Clinical trials regarding this issue are hard to conduct, since they will always deal with mothers and babies. However, we were able to find articles that dealt with mothers who had trouble breastfeeding and high risk babies because, when dealing with these groups, studies are more easily permitted, since they follow a principle of benefit to health of sample individuals. These studies could not be included in the analysis due to exclusion criteria adopted.

Therefore the cohort studies were the most common type used to evaluate factors interfering in breastfeeding duration. Periodical data collection allows a longitudinal analysis capable of measuring risk factors that reduce or increase breastfeeding duration. Only Santiago et al,.30 Nelson et al.,31 Vogel et al.41 and Mascarenhas et al.43 did not perform a survival analysis, describing only multivariate analysis with multiple logistic regressions. Despite presenting the same risk relation between pacifier use and duration of breastfeeding, these studies could have used their information better and compared it with other studies, has they carried out a survival analysis.

Cross-sectional studies are important because they can involve large samples and a large amount of data, which is practically impossible when dealing with clinical trials and cohort studies, owing to their high cost. Nevertheless, cross-sectional studies are recommended for diagnosis of the situation at the present time, but are not suitable for future predictions or risk estimates for populations.

When dealing with pacifier use and its association with duration of breastfeeding it is possible to measure percentiles of use and defined lengths of breastfeeding based on recall information collected from the mother. This is only possible because weaning is considered a key moment in the mother's life and they always remember the month when it happened. Silveira and Lamounier45 and Minagawa et al. 40 in survival analyses of cross-sectional data, establish risk estimates significantly similar to the ones obtained form cohort studies. The other authors of cross-sectional studies extrapolate their analysis methods using univariate and multivariate analysis, multiple logistic regressions and estimating risk using prevalence and odds ratios. 29,46

The present review establishes clearly the role of pacifier use as a determining factor for duration of breastfeeding, since the selected studies, regardless of design, show extremely significant levels of association even when controlled for confounding factors. Accordingly, most of the studies acknowledge the effects of complementary feeding on reducing breastfeeding duration, mainly when bottles are used.35-40 It is believable that the introduction of other foods influences the child to ask less often for the mother's breast, also to reach satiation quicker and to abandon breastfeeding early, even when being fed with an inadequate diet. However, the explanation, whether physiological or behavioral, for the reduction of breastfeeding duration through use of a pacifier remains inconclusive. In an attempt to explain this, three hypotheses can be suggested.

The first claims that the pacifier does not have a direct biological effect on the child; instead, it would be a marker of difficulty, inability or insecurity on the part of the mother with regard to breastfeeding. 25,35 The second hypothesis focuses on the pacifier acting as a method for suppressing crying and concealing difficulties experienced by the mother. This hypothesis argues that, in reality, a nipple confusion takes place. This may be due to mechanical differences and different sucking patterns established by the child for the mother's nipple and the teat of the bottle. 47 This phenomenon would lead the child to opt for sucking artificial nipples instead of the mother's.

The third, solely based on breastfeeding physiology, advocates the hypothesis that constant use of artificial nipples would diminish the previously intense stimulation of mother's nipple, consequently reducing the prolactin reflex and milk production. ${ }^{2}$ Thus, weaning would be triggered by a decrease in milk production; on the other hand, Emmerich et al. ${ }^{9}$ argue that pacifiers can generate a sense of satiation through the suction stimulus, salivary flow and deglutition, which saturate the functional system of hunger, entering into competition with it. It is necessary consider the possibility that these various factors act to reduce breastfeeding. Given these propositions, it would be appropriate to carry out further studies to test each of the hypotheses and draw up a theoretical model capable of explaining the real influence of pacifier use on breastfeeding 
duration.

This study considers it a slight possibility that it was not possible to find articles that present a nonsignificant association between pacifier use and breastfeeding duration, because references cited in the articles under review were also analyzed, with a view to making the review more thorough and avoiding bias selection. After exhaustive research, only Audi et al. 46 mentioned that Perez-Escamilla et al. 48 found a non-significant association between pacifier use and breastfeeding duration. However, more in-depth analysis revealed that Perez-Escamilla et al. 48 did not investigate the relation between pacifier use and breastfeeding duration, but rather the influence of a cloth or rag soaked or boiled in herbal infusions placed in the infant's mouth (a method called "chupón", which is commonly used in Honduras) on breastfeeding duration.

This systematic review suggests the need for standardization of methods for any further studies to be carried out, to ensure that it is possible to synthesize the results in a meta-analysis. Based on the majority of the studies found, subsequent studies on breastfeeding should be designed as prospective cohorts, calculating sample sizes in advance and following up for six months when assessing exclusive breastfeeding and 12 or 24 months when testing variables relating to breastfeeding duration. As for data collection, structured interviews conducted face to face or by telephone preformed by trained researchers should be done every six months up to

\section{Referências}

1. WHO (World Health Organization). The optimal duration of exclusive breast feeding: results of a WHO systematic review. Indian Pediatr. 2001; 38: 565-7.

2. Victora CG, Tomasi E, Olinto MTA, Barros FC. Use of pacifiers and breastfeeding duration. Lancet. 1993; 341: 404-6.

3. Fleming PM, Blair PS, Pollard K, Platt MW, Leach C, Smith I, Golding J. Pacifier use and sudden infant death syndrome results from the CESDI/SUDI case control study. Arch Dis Child. 1999; 81: 112-6.

4. Sprott J. Cot death controversies. S Afr Med J. 2006; 96 : 568.

5. Carbajal R, Veerapen S, Couderc S, Jugie M, Ville Y. Analgesic effect of breast feeding in term neonates: randomised controlled trial. BMJ. 2003; 326: 13-8.

6. Post CLA, Victora CG, Valente JG, Leal MC, Niobey FML, Sabroza PC. Fatores prognósticos de letalidade hospitalar por diarréia ou pneumonia em menores de um ano de idade: estudo de caso e controle. Rev Saúde Pública. 1992; 26 : 369-78.
24 months.

Regarding variables of interest, pacifier use up to the first 30 days should be dichotomized, while the others should be collected in a simplified way, always dichotomized when qualitative and always numerical when quantitative, avoiding use of intervals that can complicate the comparability of results. The Survival Analysis with Cox Regression using an adjusted Hazards Ratio as an estimator should be the method chosen for statistical analysis, always presenting significance values, risk calculations and their respective confidence intervals.

\section{Conclusions}

The use of a pacifier was unanimously defined as a risk factor for the reduction of breastfeeding duration, exclusive or not. However, the mechanisms by which pacifiers act on the reduction of breastfeeding duration still remain obscure.

Several factors, relating to the mother, the child, the father and hospital care, interfere with the duration of breastfeeding, with the result that this is a complex phenomenon that should be studied in all its dimensions.

Future studies assessing length of breastfeeding should follow a standardization of any methodological elements to allow for the synthesizing of scientific knowledge of this phenomenon.

7. Escuder MML, Venancio SI, Pereira JCR. Estimativa de impacto da amamentação sobre a mortalidade infantil. Rev Saúde Pública. 2003; 37: 319-25.

8. Kramer MS, Barr RG, Dagenais S, Yang H, Jones P, Ciofani L, Jané F. Pacifier use, early weaning and cry/fuss behavior. JAMA. 2001; 286:322-6.

9. Emmerich AO, Fonseca L, Elias AM, Medeiros UV. The relationship between oral habits, oronasopharyngeal alterations, and malocclusion in preschool children in Vitória, Espírito Santo, Brazil. Cad Saúde Pública. 2004; 20: 68997.

10. Moss ML, Salentijn L. The primary role of functional matrices in facial growth. Am J Orthod. 1969; 55: 566-77.

11. Brasil. Senado. Lei n. 11265 de 03 de janeiro de 2006. Regulamenta a comercialização de alimentos para lactentes e crianças de primeira infância e também a de produtos de puericultura correlatos. Diário Oficial República Federativa do Brasil, Brasília, DF, 04 jan. 2006. [acesso em: 13 dez. 2006]. Disponível em: <Http://www6.senado.gov.br/sicon/ listareferencias .action? codigobase $=2 \&$ codigodocumento $=253662>$ 
12. Ullah S, Griffiths P. Does the use of pacifiers shorten breastfeeding duration in infants? Br J Comm Nur. 2003; 8: 458-63.

13. Görbe E, Kohalmi B, Gaál G, Szánthó A, Rigó J, Harmath A, Csabay L, Szabó G. The relationship between pacifier use, bottle feeding and breast feeding. J Matern Fetal Neonatal Med. 2002; 12: 127-31.

14. Levy SM, Slager SL, Warren JJ, Levy BL, Nowak AJ. Associations of pacifier use, digit sucking, and child care attendance with cessation of breastfeeding. J Fam Pract. 2002; $51: 465$.

15. Marques NM, Lira PIC, Lima MC, Silva NL, Batista Filho M, Sharon RA, Ashworth A. Breastfeeding and early weaning practices in Northeast Brazil: a longitudinal study. Pediatrics. 2001; 108: 66-72.

16. Binns CW, Scott JA. Using pacifier: what are breastfeeding mothers doing? Breastfeed Rev. 2002; 10: 21-5.

17. Baker L. The relationship between pacifier usage in infants and breastfeeding success. Pediatr Nurs. 2000; 26: 402-4.

18. Righard L. Are breastfeeding problems related to incorrect breastfeeding technique and the use of pacifiers and bottles? Birth. 1998; 25: 40-4.

19. Righard L, Alade MO. Breastfeeding and use of pacifiers. Birth. 1997; 25: 40-4.

20. Kloeblen-Tanver AS. Pacifier use is associated with shorter breastfeeding duration among low-income women. Pediatrics. 2001; 108: 526.

21. Centuori S, Burmaz T, Ronfani L, Frangiacomo M, Quintero S, Pavan C, Davanzo R, Cattaneo A. Nipple care, sore nipples, and breastfeeding: a randomized trial. J Hum Lact. 1999; 15: 127-32.

22. Barros FC, Victora, CG, Morris SS, Halpern R, Horta BL, Tomasi E. Breastfeeding, pacifier use and infant development at 12 months of age: a birth cohort study in Brazil. Paediatr Perinat Epidemiol. 1997; 11: 441-50.

23. Collins CT, Ryan P, Crowther CA, Mcphee AJ, Paterson S, Hiller JE. Effect of bottles, cups, and dummies on breast feeding in preterm infants: a randomized controlled trial. BMJ. 2004; 329: 193-8.

24. Cunha AJ, Leite AM, Machado MM. Breastfeeding and pacifier use in Brazil. Indian J Pediatr. 2005; 72: 209-12.

25. Dewey KG, Nommsen-Rivers LA, Heinig MJ, Cohen RJ. Risk factors for suboptimal infant breastfeeding behavior, delayed onset of lactation, and excess neonatal weight loss. Pediatrics. 2003; 112: 607-9.

26. Hörnell A, Aarts C, Kylberg E, Hofvander Y, Gebre-Medhin M. Breastfeeding patterns in exclusively breastfed infants: a longitudinal prospective study in Uppsala, Sweden. Acta Pædiatr. 1999; 88: 203-11.

27. Aarts C, Hörnell A, Kylberg E, Hofvander Y, GebreMedhin M. Breastfeeding patterns in relation to thumb sucking and pacifier use. Pediatrics. 1999; 104: 50-60.

28. Kelmanson IA. Use of a pacifier and behavioural features in 2-4-month-old infants. Acta Pædiatr. 1999; 88: 1258-61.

29. Mikiel-Kostyra K, Mazur J, Wojdan-Godek E. Factors affecting exclusive breastfeeding in Poland: cross-sectional survey of population-based samples. Soz-Präventivmed. 2005; 50: 52-9.
30. Santiago LB, Bettiol H, Barbieri MA, Guttierrez MRP, Del Ciampo LA. Incentivo ao aleitamento materno: a importância do pediatra com treinamento específico. J Pediatr (Rio J). 2003; 79: 504-12.

31. Nelson EA, Yu LM, Williams S. International child care practices: breastfeeding and pacifier use. J Hum Lact. 2005; 21: 289-95

32. Cotrim LC, Venancio SI, Escuder MML. Uso de chupeta e amamentação em crianças menores de quatro meses no estado de São Paulo. Rev Bras Saude Matern Infant. 2002; 2: 245-52.

33. Vieira GO, Almeida JAG, Silva LR, Cabral VA, SantanaNetto PV. Fatores associados ao aleitamento materno e desmame em Feira de Santana, Bahia. Rev Bras Saúde Matern Infant. 2004; 4: 143-50.

34. Vieira GO, Silva LR, Vieira TO, Almeida JAG, Cabral VA. Hábitos alimentares de crianças menores de um ano amamentadas e não-amamentadas. J Pediatr (Rio J). 2004; 80: 411-6.

35. Howard CR, Howard FM, Lanphear B, Eberly S, deBlieck EA, Oakes D, Lawrence RA. Randomized clinical trial of pacifier use and bottle-feeding or cupfeeding and their effect on breastfeeding. Pediatrics. 2003; 111: 511-8.

36. Victora CG, Behague DP, Barros FC, Olinto MTA, Weiderpass E. Pacifier use and short breastfeeding duration: cause, consequence, or coincidence? Pediatrics. 1997; 99: 445-53.

37. Riva E, Banderali G, Agostoni C, Silano M, Radaelli G, Giovannini M. Factors associated with initiation and duration of breastfeeding in Italy. Acta Pædiatr. 1999; 88: 411-5.

38. Soares MEM, Giugliani ERJ, Braun ML, Salgado ACN, Oliveira AP, Aguiar PR. Uso de chupeta e sua relação com o desmame precoce em população de crianças nascidas em hospital amigo da criança. J Pediatr (Rio J). 2003; 79: 30916.

39. Giovannini M, Riva E, Banderali G, Di Giacomo S, Veehof S, Sala M, Radaelli G, Agostoni C. Feeding practices of infants through the first year of life in Italy. Acta Pædiatr. 2004; 93: 492-7.

40. Minagawa AT, Oliveira IMV, Fujimori E, Laurenti D, Montero MRJM. Perfil do aleitamento materno em menores de dois anos na cidade de Itupeva, SP, Brasil. Arq Latinoam Nutr. 2005; 55: 132-9.

41. Vogel AM, Hutchison BL, Mitchell EA. The impact of pacifier use on breastfeeding: a prospective cohort study. J Paediatr Child Health. 2001; 37: 58-63.

42. Scott JA, Binns CW, Oddy WH, Graham KI. Predictors of breastfeeding duration: evidence from a cohort study. Pediatrics. 2006; 117: 646-55.

43. Mascarenhas MLW, Albernaz EP, Silva MB, Silveira RB. Prevalência de aleitamento materno exclusivo nos três primeiros meses de vida e seus determinantes no sul do Brasil. J Pediatr (Rio J). 2006; 82: 289-94.

44. Howard CR, Howard FM, Lanphear B, deBlieck EA, Eberly S, Lawrence RA. The effects of early pacifier use on breastfeeding duration. Pediatrics. 1999; 103: 33-8. 
45. Silveira FJF, Lamounier JA. Fatores associados à duração do aleitamento materno em três municípios na região do alto Jequitinhonha, Minas Gerais, Brasil. Cad Saúde Pública. 2006; 22: 69-77.

46. Audi CAF, Correa AMS, Latorre MRDO. Alimentos complementares e fatores associados ao aleitamento materno e ao aleitamento materno exclusivo em lactentes até 12 meses de vida em Itapira, São Paulo, 1999. Rev Bras Saúde Matern Infant. 2003; 3: 85-93.

Recebido em 29 de junho de 2007

Versão final apresentada em 23 de outubro de 2008

Aprovado em 31 de outubro de 2008
47. Neifert M, Lawrence RA, Seacat J. Nipple confusion: toward a formal definition. J Pediatr. 1995; 126: S125-S9.

48. Perez-Escamilla R, Segura-Millan S, Canahuati J, Allen H. Prelacteal feeds are negatively associated with breastfeeding outcomes in Honduras. J Nutr. 1996; 126: 2765-73. 